Bundesgesundheitsbl 2020 - 63:1297-1298 https://doi.org/10.1007/s00103-020-03129-4 Online publiziert: 7. April 2020

(c) Springer-Verlag GmbH Deutschland, ein Teil von Springer Nature 2020
Sophie-Charlotte Götz ${ }^{1}$ Georg Marckmann' · Joerg Hasford ${ }^{2}$. Ralf J. Jox ${ }^{3,4}$ (D)

'Institut für Ethik, Geschichte und Theorie der Medizin, Ludwig-Maximilians-Universität München, München, Deutschland

${ }^{2}$ Institut für Med. Informationsverarbeitung, Biometrie und Epidemiologie, Ludwig-MaximiliansUniversität München, München, Deutschland

${ }^{3}$ Unité d'Éthique Clinique, Universitätsklinikum Lausanne (CHUV), Universität Lausanne, Lausanne, Schweiz

${ }^{4}$ Institut des Humanités en Médecine, Universitätsklinikum Lausanne (CHUV), Universität Lausanne, Lausanne, Schweiz

\section{Erratum zu: Arzneimittelprüfung an nicht einwilligungsfähigen Erwachsenen: Kritische Bewertung der neuen gesetzlichen Regelung durch medizinische Ethikkommissionen in Deutschland}

Erratum zu:

Bundesgesundheitsbl 2019

https://link.springer.com/article/10.1007/

s00103-019-03058-x

In der Originalpublikation des Artikels ist ein Fehler in der Methodendarstellung im Text und in der Zusammenfassung und in der veröffentlichten $\bullet$ Tab. 2 aufgetreten.

Innerhalb des Methodenteils wurde fälschlicherweise von einer Gesamtheit von 53 Ethikkommissionen berichtet. Korrekt sind es 52 Ethikkommissionen.

In - Tab. 2 ist ein Rechenfehler enthalten. Die korrigierte Version ist im Folgenden abgebildet.

\section{Korrespondenzadresse}

Ralf J. Jox

Institut des Humanités en Médecine,

Universitätsklinikum Lausanne (CHUV),

Universität Lausanne

Avenue de Provence 82, 1007 Lausanne,

Schweiz

ralf.jox@chuv.ch 


\section{Erratum}

Tab. 2 Einordnung fünf fiktiver Forschungsstudien hinsichtlich ihres Nutzenpotenzials (in die Kategorien eigennütziger, ausschließlich gruppennütziger und rein fremdnütziger Forschung), Anzahl gültiger Stimmen $=84$

Studienbeispiel

Eigennützig Ausschließlich gruppennützig

Rein fremd- Weiß

Keine

$n(\%)$

nützig nicht

Angabe

Biomarkerbestimmung, die Erkenntnisse zum allgemeinen Krankheitsgeschehen der Schizophrenie verspricht

Studienbedingte Gabe eines herzunterstützenden Medikaments bei 69 (82) Patienten mit Alzheimerdemenz und Herzschwäche

$2(2)$

$74(88)$

$4(5)$

$4(5)$

$0(0)$

Teilnahme an einer Beobachtungsstudie zur ärztlichen Kommunika-

$69(82) \quad 6(7)$

$4(5)$

$4(5)$

$1(1)$

tion bei Gesprächen mit Demenzpatienten

$17(20)$

$50(60)$

$12(14)$

$3(4)$

$2(2)$

Beobachtende Bildgebungsstudie zur Kontrolle eines neuen OP-Verfahrens bei Prostatektomie, das Vorteile gegenüber den herkömmli-

$30(36)$

$36(43)$

$12(14)$

$4(5)$

$2(2)$

chen Verfahren verspricht

Evaluationsstudie eines Mobilisierungsplans nach Hüft-TEP mit dem

$49(58)$

$28(33)$

$4(5)$

$2(2)$

$1(1)$ 